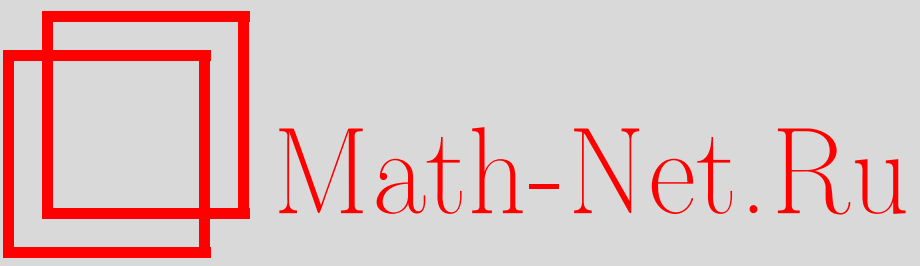

С. В. Клименко, И. Н. Никитин, Исследование особенностей на мировых листах открытых релятивистских струн, ТМФ, 1998, том 114, номер 3, 380-398

DOI: https://doi.org/10.4213/tmf847

Использование Общероссийского математического портала Math-Net.Ru подразумевает, что вы прочитали и согласны с пользовательским соглашением

http://www.mathnet.ru/rus/agreement

Параметры загрузки:

IP: 3.91 .87 .62

26 апреля 2023 г., 13:01:47 
ТЕОРЕТИЧЕСКАЯ

И МАТЕМАТИЧЕСКАЯ

ФИЗИКА

Том 114, № 3

март, 1998

(C) 1998 г.

С. В. Клименко*, И.Н. Никитин*

\section{ИССЛЕДОВАНИЕ ОСОБЕННОСТЕЙ НА МИРОВЫХ ЛИСТАХ ОТКРЫТЫХ РЕЛЯТИВИСТСКИХ СТРУН}

Проведена классификация устойчивых особых точек на мировых листах открытых релятивистских струн.

\section{ВВЕДЕНИЕ}

Вопросами устойчивости особых точек на поверхностях, получаемых гладкими отображениями $\mathbf{R}^{2}$ в $\mathbf{R}^{d}$, занимается теория особенностей гладких отображений [1]. При $d=3$ устойчивыми $^{1)}$ особенностями являются изолированные точки пинча (в окрестности которых поверхность имеет вид зонтика Уитни), при $d>3$ поверхность в общем положении погружена в $\mathbf{R}^{d}$.

Иным поведением обладают особенности мировых листов струн - поверхностей экстремальной плошади в $d$-мерном пространстве Минковского. Впервые этот вопрос рассматривался в работе [2] для мировых листов замкнутых струн в 3-мерном пространстве Минковского. Было показано, что все такие поверхности особые, причем особенностями в обшем положении являются линии на мировом листе, вдоль которых на сечениях мирового листа плоскостями постоянного времени расположены точки возврата. В [2] отмечалась связь таких особенностей с сингулярными решениями двумерного уравнения Лиувилля, исследованными ранее в работах [3].

Цель данной работы - детальная классификация особенностей на мировых листах открытых струн. В разделе 1 мы покажем, что устойчивые особенности мировых листов

\footnotetext{
1) Устойчивыми называются особые точки, сохраняюшиеся при малых деформациях поверхности. Устойчивыми являются также линии самопересечений поверхности в $\mathbf{R}^{3}$ и точки самопересечений в $\mathbf{R}^{4}$. Топологические свойства самопересечений поверхностей общего вида и поверхностей, исследуемых в теории струны, совпадают и в данной работе не рассматриваются.
}

* Институт физики высоких энергий, Протвино, Московская обл., Россия; German National Center for Information Technology, Germany 
при $d=3$ - линии, при $d=4$ - точки. При $d>4$ мировой лист в общем положении является погруженным. В остальных разделах описана глобальная структура особенностей на мировых листах открытых струн.

Сушествование таких особенностей на струнах интересно с физической точки зрения.

1. Особенности имеют вид изломов на струне, в которых линейная плотность энергии-импульса струны стремится к бесконечности. "Идеальная" теория струн работает и в таком режиме, но струнные модели адронов вблизи особых точек выходят за пределы своей применимости [4].

2. В наиболее последовательной модели разрыва струн [5] (основанной на минимизации действия Намбу-Гото в топологическом классе "лента с полубесконечным разрезом") положение точки разрыва не определяется начальными данными, а является свободным параметром. Фактически эта модель утверждает, что разрыв струны может произойти в произвольной точке, в дальнейшем продукты распада распространяются по законам движения свободных струн, в целом же полученный мировой лист является экстремальной поверхностью. При использовании этой модели для описания распада адронов ее необходимо дополнить конкретным механизмом разрыва струн. Физически правдоподобной выглядит гипотеза о разрыве струн по особым точкам. При этом массы и спины продуктов распада полностью определяются динамикой струны ${ }^{2}$.

3. Поскольку особенности на струне проявляют себя как устойчивые образования внутри адронов, в окрестности которых сконцентрирован значительный импульс, возможна физическая интерпретация особенностей как элементов структуры экзотических (гибридных) адронных состояний. Преимущественный разрыв струны в особых точках может являться причиной нестабильности этих состояний.

4. Для описания данных явлений на квантовом уровне можно использовать методы квантования частных классов движения струн в некритической размерности, разработанные в [6].

\section{1. МИРОВОЙ ЛИСТ ОТКРЫТОЙ СТРУНЫ}

Пусть в $d$-мерном пространстве Минковского задана периодическая светоподобная кривая $Q_{\mu}(\sigma)$ :

$$
Q^{\prime 2}(\sigma)=0, \quad Q_{\mu}(\sigma+2 \pi)=Q_{\mu}(\sigma)+2 P_{\mu}
$$

$2 P_{\mu}$ - период кривой. Будем называть эту кривую опорной.

\footnotetext{
${ }^{2)}$ Следует также обратить внимание на отмеченную в [5] связь процессов разрыва и особенностей на струнах: при разрыве струны в неособой точке вследствие мгновенного изменения граничных условий на продуктах распада появляются особенности. В то же время при разрыве струны в особой точке дополнительные особенности на мировом листе не появляются (поскольку режимы в особой точке и на образующихся свободных концах совпадают).
} 

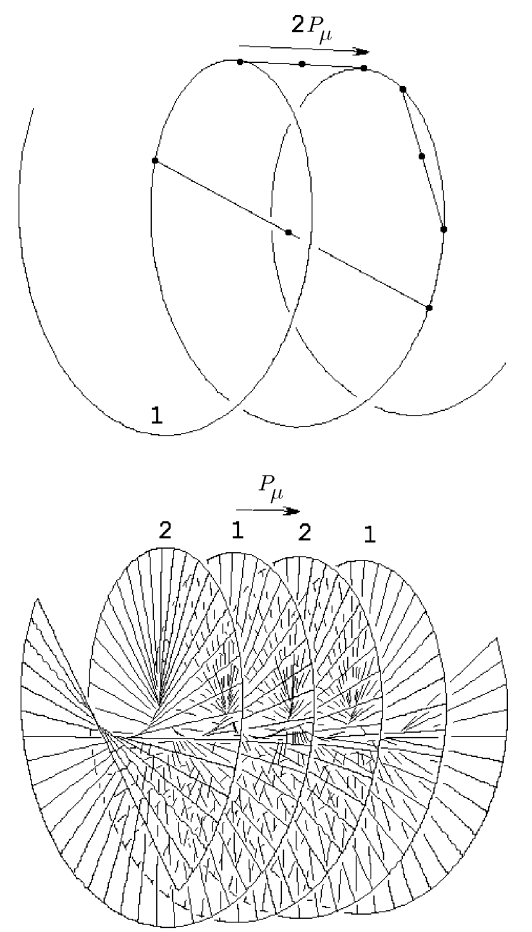

Рис. 1. Опорная кривая и мировой лист струны.

Мировой лист открытой струны (рис. 1) строится как геометрическое место середин отрезков, соединяющих всевозможные пары точек на опорной кривой

$$
x_{\mu}\left(\sigma_{1}, \sigma_{2}\right)=\frac{1}{2}\left(Q_{\mu}\left(\sigma_{1}\right)+Q_{\mu}\left(\sigma_{2}\right)\right)
$$

Опорная кривая связана с начальными данными (координатами и плотностями импульсов струны $x_{\mu}(\sigma), p_{\mu}(\sigma)$, заданными в произвольном сечении мирового листа $\left(\sigma_{1}(\sigma), \sigma_{2}(\sigma)\right), 0 \leq \sigma \leq \pi$, и продолженными на всю ось $\sigma$ как четные и $2 \pi$-периодические функции):

$$
Q_{\mu}(\sigma)=x_{\mu}(\sigma)+\int_{0}^{\sigma} d \sigma^{\prime} p_{\mu}\left(\sigma^{\prime}\right) \Leftrightarrow\left\{\begin{array}{l}
x_{\mu}(\sigma)=\left(Q_{\mu}(\sigma)+Q_{\mu}(-\sigma)\right) / 2 \\
p_{\mu}(\sigma)=\left(Q_{\mu}^{\prime}(\sigma)+Q_{\mu}^{\prime}(-\sigma)\right) / 2
\end{array}\right.
$$

Мировой лист имеет два светоподобных края: опорная кривая 1 и ее образ при сдвиге на полупериод 2 (см. рис. 1). Мировой лист переходит в себя при сдвиге на $2 P_{\mu}$. При сдвиге на $P_{\mu}$ он также переходит в себя, но при этом меняются местами его края. Сечение мирового листа плоскостью постоянного времени дает струну в этот момент времени. Доказательство этих фактов вынесено в приложение 1. 
Поверхность (1) имеет два края. Первый край совпадает с опорной кривой, второй получается из опорной кривой сдвигом на полупериод:

$$
x_{\mu}(\sigma, \sigma)=Q_{\mu}(\sigma), \quad x_{\mu}(\sigma, \sigma+2 \pi)=Q_{\mu}(\sigma)+P_{\mu} .
$$

Края мирового листа светоподобны. Кроме того, любой вектор из касательной плоскости к мировому листу на его крае ортогонален касательной к краю $Q_{\mu}^{\prime}$. (Для доказательства необходимо разложить (1) в окрестности края до членов 2-го порядка и использовать тождество $Q^{\prime} Q^{\prime \prime}=\left(Q^{\prime 2}\right)^{\prime} / 2=0$.) Эта плоскость касается светового конуса по направлению $Q_{\mu}^{\prime}$. Мы будем называть такие плоскости изотропными.

Края мирового листа являются мировыми линиями концов струны. Сечение мирового листа плоскостью постоянного времени дает струну в этот момент времени. Касательная к струне $l_{\mu}$ содержится в касательной плоскости к мировому листу, поэтому на крае она ортогональна $Q_{\mu}^{\prime}$ :

$$
l_{\mu} Q_{\mu}^{\prime}=0, \quad l_{0}=0 \Rightarrow \vec{l} \vec{Q}^{\prime}=0
$$

Таким образом, концы струны движутся со скоростью света под прямым углом к направлению струны в этой точке.

Полный импульс струны равен полупериоду опорной кривой $P_{\mu}$ (см. приложение 1$)$. Проекция опорной кривой в подпространство, ортогональное $P_{\mu}$ (система покоя струны), является замкнутой кривой $\vec{Q}(\sigma)$. Опорную кривую можно восстановить по ее проекции:

$$
Q_{0}^{\prime}=\left|\vec{Q}^{\prime}\right| \Rightarrow Q_{0}\left(\sigma_{1}\right)-Q_{0}(0)=\int_{0}^{\sigma_{1}}\left|\vec{Q}^{\prime}(\sigma)\right| d \sigma=L\left(\sigma_{1}\right),
$$

$L(\sigma)$ - длина дуги вдоль кривой $\vec{Q}(\sigma)$ между точками $\vec{Q}(0)$ и $\vec{Q}(\sigma)$. Полная длина кривой $\vec{Q}(\sigma)$ равна удвоенной массе струны $L(2 \pi)=2 \sqrt{P^{2}}$. Кривую $\vec{Q}(\sigma)$ можно параметризовать ее длиной $\sigma=\pi L / \sqrt{P^{2}}$, тогда $Q_{0}^{\prime}=\left|\vec{Q}^{\prime}\right|=\sqrt{P^{2}} / \pi$. Эту параметризацию мы будем называть калибровкой системы покоя (КСП).

Данное представление мирового листа удобно для исследования особых точек на нем. Прежде всего, почему такие точки появляются? Касательными векторами к мировому листу в точке $x_{\mu}\left(\sigma_{1}, \sigma_{2}\right)$ являются $Q_{\mu}^{\prime}\left(\sigma_{1}\right)$ и $Q_{\mu}^{\prime}\left(\sigma_{2}\right)$, т.е. касательные векторы к опорной кривой в соответствующих точках. Если эти два вектора линейно независимы, то они определяют касательную плоскость к мировому листу, и данная точка является регулярной (точка 1 на рис. 2а). В противном случае, если эти векторы параллельны, точка является особой ${ }^{3)}$. Исключая тривиальный случай точки 2 , когда она лежит на

\footnotetext{
3) Это условие необходимо, для того чтобы рассматриваемая точка была особой. Далее мы покажем, что для поверхностей (1) оно также является достаточным.
} 


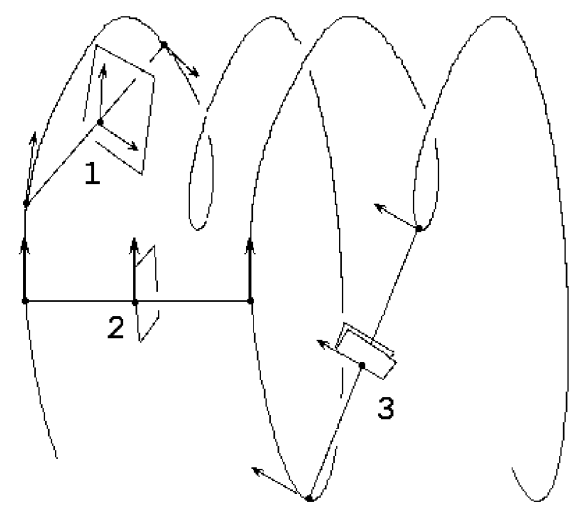

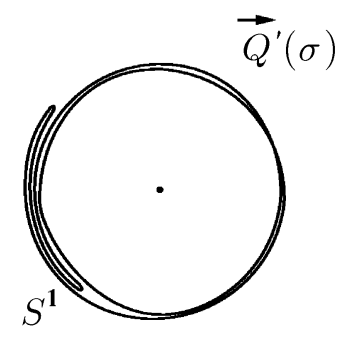

$\sigma$

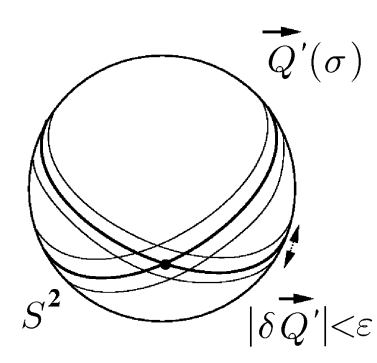

B

Рис. 2. Особые точки мирового листа.

крае мирового листа, мы будем исследовать внутренние особые точки 3. Для этого необходимо проанализировать разложения Тейлора мирового листа вблизи особых точек и принять во внимание некоторые топологические свойства опорной кривой.

Прежде всего отметим два важных свойства особых точек.

1. Случай соответствующей точки 3 на рис. 2 а устойчив в пространстве Минковского размерностей 3 и 4 и неустойчив при бо́льших значениях размерности.

ДоКАЗАТЕЛЬСТво. В КСП условие сушествования особой точки принимает вид равенства единичных касательных векторов к кривой $\vec{Q}(\sigma)$ :

$$
Q_{\mu}^{\prime}\left(\sigma_{1}\right) \| Q_{\mu}^{\prime}\left(\sigma_{2}\right), \quad Q_{0}^{\prime}\left(\sigma_{1}\right)=Q_{0}^{\prime}\left(\sigma_{2}\right)=\sqrt{P^{2}} / \pi \Rightarrow \vec{Q}^{\prime}\left(\sigma_{1}\right)=\vec{Q}^{\prime}\left(\sigma_{2}\right)
$$

В $d$-мерном пространстве Минковского кривая $\vec{Q}^{\prime}(\sigma)$ лежит на $(d-2)$-мерной сффере и может обладать устойчивыми самопересечениями только при $d=3$ (на рис. $2 б$ кривая лежит на окружности, множество решений уравнения $\vec{Q}^{\prime}\left(\sigma_{1}\right)=\vec{Q}^{\prime}\left(\sigma_{2}\right)$ одномерно) и $d=4$ (на рис. 2в кривая лежит на 2-мерной сфере, устойчивые самопересечения располагаются в изолированных точках). 
Таким образом, устойчивые особенности на мировых листах существуют только при значениях размерности 3 (линии) и 4 (точки). Такие особенности не устраняются при малых деформациях мирового листа (в классе экстремальных поверхностей), которые соответствуют малым деформациям опорной кривой, или эквивалентно начальных данных ${ }^{4)}$

2. Линейная плотность энергии-импульса струны определяется формулой

$$
\frac{d \mathcal{P}_{\mu}}{d l}=\frac{p_{\mu}}{\sqrt{-x^{\prime 2}}}=\frac{Q_{\mu}^{\prime}\left(\sigma_{1}\right)+Q_{\mu}^{\prime}\left(\sigma_{2}\right)}{\sqrt{-\left(Q^{\prime}\left(\sigma_{1}\right)-Q^{\prime}\left(\sigma_{2}\right)\right)^{2}}} \quad\left(d \mathcal{P}_{\mu}=p_{\mu} d \sigma, \quad d l=\sqrt{d \vec{x}^{2}}, \quad d x_{0}=0\right)
$$

Векторы $Q^{\prime}\left(\sigma_{1}\right)$ и $Q^{\prime}\left(\sigma_{2}\right)$ светоподобны, в особых точках (и на крае листа) они параллельны, поэтому в этих точках $d \mathcal{P}_{\mu} / d l \rightarrow \infty$.

\section{2. ОСОБЕННОСТИ НА МИРОВЫХ ЛИСТАХ ПРИ $d=3$ - ЛИНИИ КИНКОВ}

При $d=3$ пространство, ортогональное импульсу $P_{\mu}$, двумерно. Гладкие замкнутые кривые на плоскости разбиваются на классы по числу оборотов $\nu$ единичного касательного вектора. Для вылуклльх кривых (не имеющих точек перегиба) полярный угол единичного касательного вектора зависит от $\sigma$ монотонно. Поэтому для каждого направления касательной найдутся $\nu-1$ точек с тем же направлением касательной. Таким образом, на струне в $d=3$ постоянно существуют $\nu-1$ особых точек. Особые точки на мировом листе образуют линии. Касательные к ним светоподобны:

$$
d x_{\mu}=\frac{1}{2}\left(Q_{\mu}^{\prime}\left(\sigma_{1}\right) d \sigma_{1}+Q_{\mu}^{\prime}\left(\sigma_{2}\right) d \sigma_{2}\right) \| Q_{\mu}^{\prime}\left(\sigma_{1}\right) .
$$

Исследуем форму мирового листа в окрестности особой линии:

$$
\begin{aligned}
& x\left(\sigma_{1}+\Delta \sigma_{1}, \sigma_{2}+\Delta \sigma_{2}\right)=\frac{1}{2}\left(Q_{1}+Q_{2}\right)+\frac{1}{2} Q_{1}^{\prime}\left(\Delta \sigma_{1}+\lambda \Delta \sigma_{2}\right)+ \\
& +\frac{1}{4}\left(Q_{1}^{\prime \prime} \Delta \sigma_{1}{ }^{2}+Q_{2}^{\prime \prime} \Delta{\sigma_{2}}^{2}\right)+o\left(\Delta \sigma_{1}{ }^{2}+\Delta \sigma_{2}{ }^{2}\right), \quad Q_{2}^{\prime}=\lambda Q_{1}^{\prime} .
\end{aligned}
$$

Векторы $Q_{1}^{\prime}, Q_{1}^{\prime \prime}, Q_{2}^{\prime \prime}$ линейно зависимы, т.к. $Q_{1}^{\prime \prime}$ и $Q_{2}^{\prime \prime}$ лежат в 2-мерной плоскости, ортогональной $Q_{1}^{\prime}$, и эта плоскость содержит $Q_{1}^{\prime}$ :

$$
\begin{aligned}
Q_{2}^{\prime \prime}= & \alpha Q_{1}^{\prime}+\beta Q_{1}^{\prime \prime}, \\
x= & \frac{1}{2}\left(Q_{1}+Q_{2}\right)+\frac{1}{2} Q_{1}^{\prime}\left(\Delta \sigma_{1}+\lambda \Delta \sigma_{2}+\frac{\alpha}{2} \Delta{\sigma_{2}}^{2}\right)+ \\
& +\frac{1}{4} Q_{1}^{\prime \prime}\left(\Delta \sigma_{1}{ }^{2}+\beta \Delta{\sigma_{2}}^{2}\right)+o\left(\Delta{\sigma_{1}}^{2}+\Delta{\sigma_{2}}^{2}\right) .
\end{aligned}
$$

\footnotetext{
${ }^{4)}$ Существует $\varepsilon>0$ такое, что произвольные вариации начальных данных, ограниченные условием $\left|\delta \vec{x}^{\prime}(\sigma)\right|<\varepsilon,|\delta \vec{p}(\sigma)|<\varepsilon \forall \sigma$ (т.е. $\left|\delta \vec{Q}^{\prime}(\sigma)\right|<2 \varepsilon \forall \sigma$ в силу (2)), не устраняют особую точку на мировом листе.
}

3 Теоретическая и математическая физика, т. 114, № 3, 1998 г. 


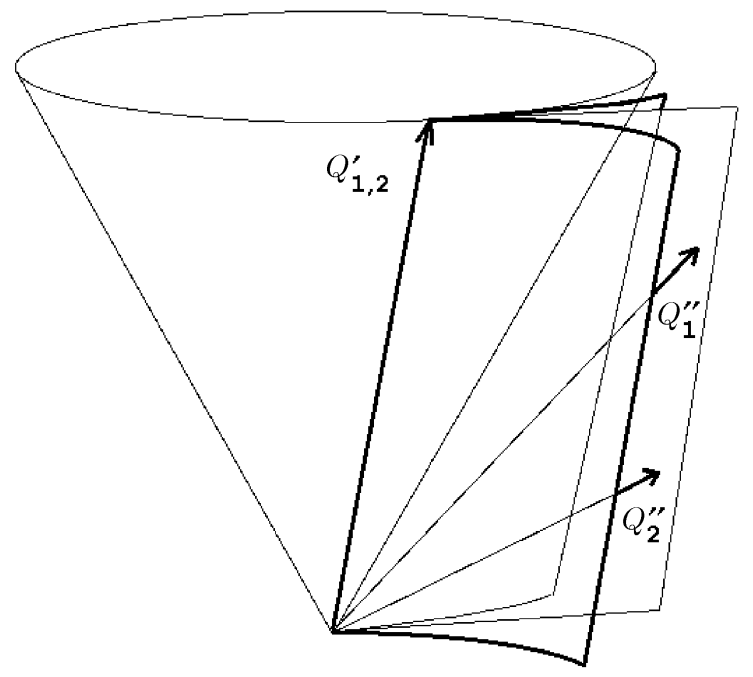

Рис. 3. Форма мирового листа вблизи особой линии.

Отображение $\left(\Delta \sigma_{1}, \Delta \sigma_{2}\right) \rightarrow(X, Y)=\left(\Delta \sigma_{1}+\lambda \Delta \sigma_{2}, \Delta \sigma_{1}{ }^{2}+\beta \Delta \sigma_{2}{ }^{2}\right)$ имеет складку на параболе $Y=X^{2} /\left(1+\lambda^{2} / \beta\right)$. Образ плоскости $\left(\Delta \sigma_{1}, \Delta \sigma_{2}\right)$ лежит вьше параболы при $\beta>-\lambda^{2}$ и ниже параболы при $\beta<-\lambda^{2}$. Таким образом, в малой окрестности точки разложения мировой лист близок к полуплоскости с границей, параллельной $Q_{1}^{\prime}$ и содержашей вектор $Q_{1}^{\prime \prime} \operatorname{sign}\left(\beta+\lambda^{2}\right)$. При переходе через особую линию мировой лист претерпевает складку (рис. 3). Части мирового листа, разделенные особой линией, имеют вблизи нее обшую касательную плоскость. Эта плоскость изотропна, как и касательная плоскость на крае.

Сечение мирового листа плоскостью постоянного времени имеет точку возврата (кинк). Направление касательной к струне в точке возврата ортогонально мировой линии кинка, т.к. касательная плоскость к мировому листу на линии кинка изотропна. Кинк движется со скоростью света под прямым углом к направлению струны в этой точке (рис. 4).

ЗАмечаниЕ. Точности разложения (3) недостаточно для исследования формы поверхости в окрестности точек с $\beta=-\lambda^{2}$. Можно показать, что величина $\beta / \lambda^{2}$ параметрически инвариантна и в КСП $\lambda=1, \beta=\varphi^{\prime}\left(\sigma_{2}\right) / \varphi^{\prime}\left(\sigma_{1}\right)$, где $\varphi(\sigma)$ - полярньй угол касательного вектора и $\varphi\left(\sigma_{1}\right)=\varphi\left(\sigma_{2}\right) \bmod 2 \pi$. Отсюда следует, что для выпуклых опорных кривых $\beta>0$, т.е. упомянутый особый случай не возникает.

Приведем некоторые свойства особых точек в $d=3$ (подробные доказательства можно найти в [7]):

1. Для выпуклых опорных кривых (см.рис. 4) кинк движется внутри струны, не достигая ее концов. 


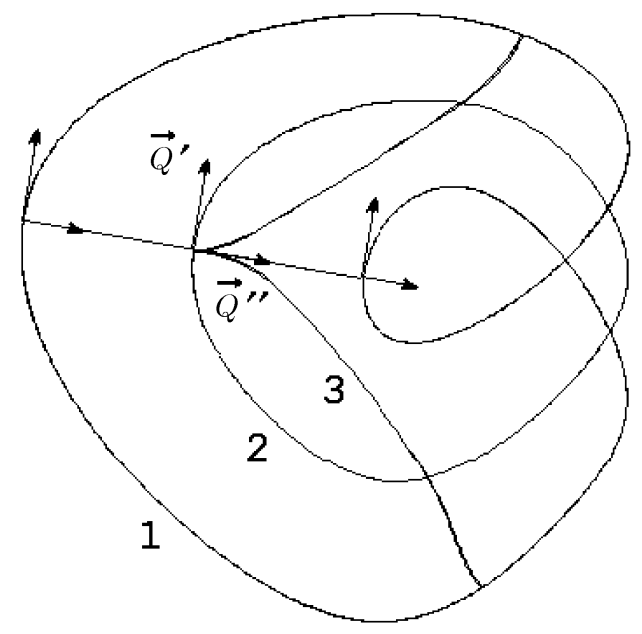

Рис. 4. Кинк на струне (система покоя): 1 - опорная кривая, 2 - линия кинка, 3 струна

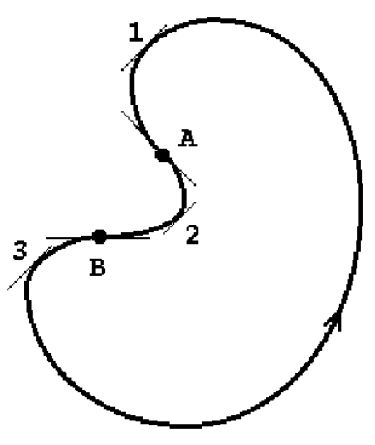

a

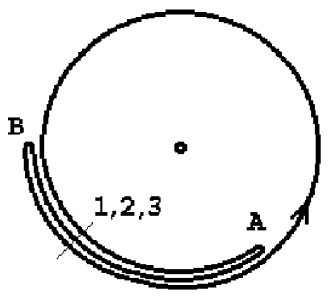

б

Рис. 5. Невыпуклая опорная кривая (а) и годографф ее единичного касательного вектора (б). А, В - точки перегиба.

2. Невыпуклые участки опорной кривой порождают новые кинки (рис. 5, касательные в точках 1, 2, 3 параллельны, поэтому на струне имеются кинки). Число точек перегиба на гладких замкнутых кривых четно.

На рис. 6 рассмотрен случай, когда опорная кривая имеет две точки перегиба. Здесь на участках a, b, с для каждой точки $\sigma_{1}$ имеется точка $\sigma_{2}$ c сонаправленной касательной. Эти точки образуют кривые на плоскости параметров $\left(\sigma_{1}, \sigma_{2}\right): \mathrm{ab}=\left\{\left(\sigma_{1}, \sigma_{2}\right): \varphi\left(\sigma_{1}\right)=\right.$ $\left.\varphi\left(\sigma_{2}\right), \sigma_{1} \in \mathrm{a}, \sigma_{2} \in \mathrm{b}\right\}$ и т.д.

Будем рассматривать сечения графика $\varphi(\sigma)$ прямой постоянного $\varphi$, двигающейся 

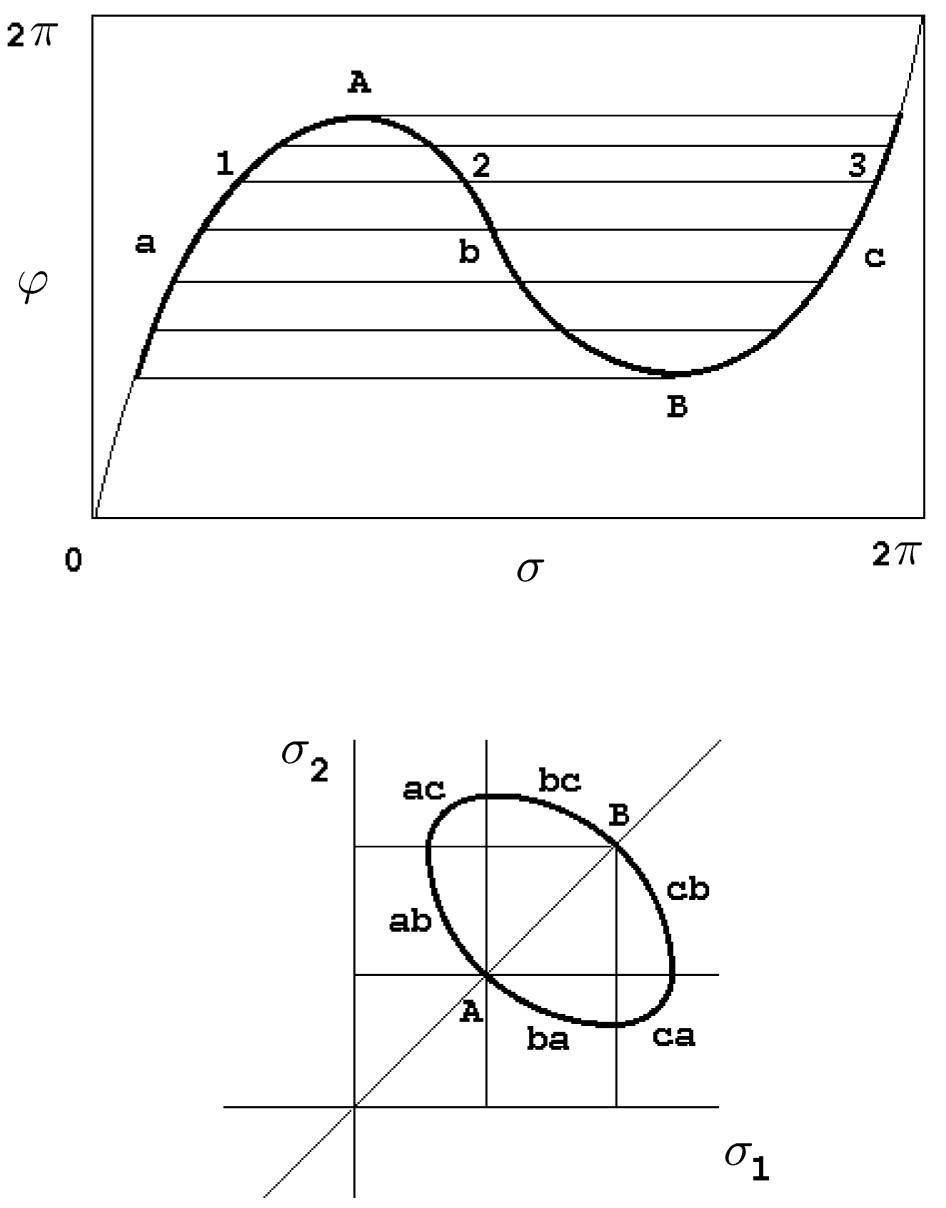

Рис. 6. Зависимость от $\sigma$ полярного угла касательного вектора.

вниз. Положим $\sigma_{1}<\sigma_{2}$. Уравнение $\varphi\left(\sigma_{1}\right)=\varphi\left(\sigma_{2}\right)$ имеет решения при $\varphi<\varphi_{\text {A. }}$ В этот момент в точке А на плоскости параметров начинается участок ab. В окрестности точки А

$$
\varphi\left(\sigma_{\mathrm{A}}+\Delta \sigma\right)=\varphi_{\mathrm{A}}+\frac{1}{2} \varphi_{\mathrm{A}}^{\prime \prime} \Delta \sigma^{2}+o\left(\Delta \sigma^{2}\right)
$$

решением уравнения $\varphi\left(\sigma_{1}\right)=\varphi\left(\sigma_{2}\right)$ в низшем приближении является $\sigma_{1}=\sigma_{\mathrm{A}}+\Delta \sigma$, $\sigma_{2}=\sigma_{\mathrm{A}}-\Delta \sigma$. Поэтому вблизи точки А участок аb ортогонален прямой $\sigma_{1}=\sigma_{2}$. При дальнейшем уменьшении $\varphi$ значение $\sigma_{1}$ уменьшается, $\sigma_{2}$ увеличивается до тех пор, пока $\varphi$ не станет равным $\varphi_{\mathrm{B}}$. В этот момент скорость изменения $\sigma_{2}$ неограниченно возрастает, скорость $\sigma_{1}$ остается ограниченной (скорость изменения $\varphi$ постоянна), поэтому вблизи $\varphi=\varphi_{\mathrm{B}}$ касательная к ab направлена вертикально, при этом $\sigma_{2}=\sigma_{\mathrm{B}}$. Теперь будем увеличивать $\varphi$. Значение $\sigma_{2}$ переходит с интервала b на интервал с. На линии ас 

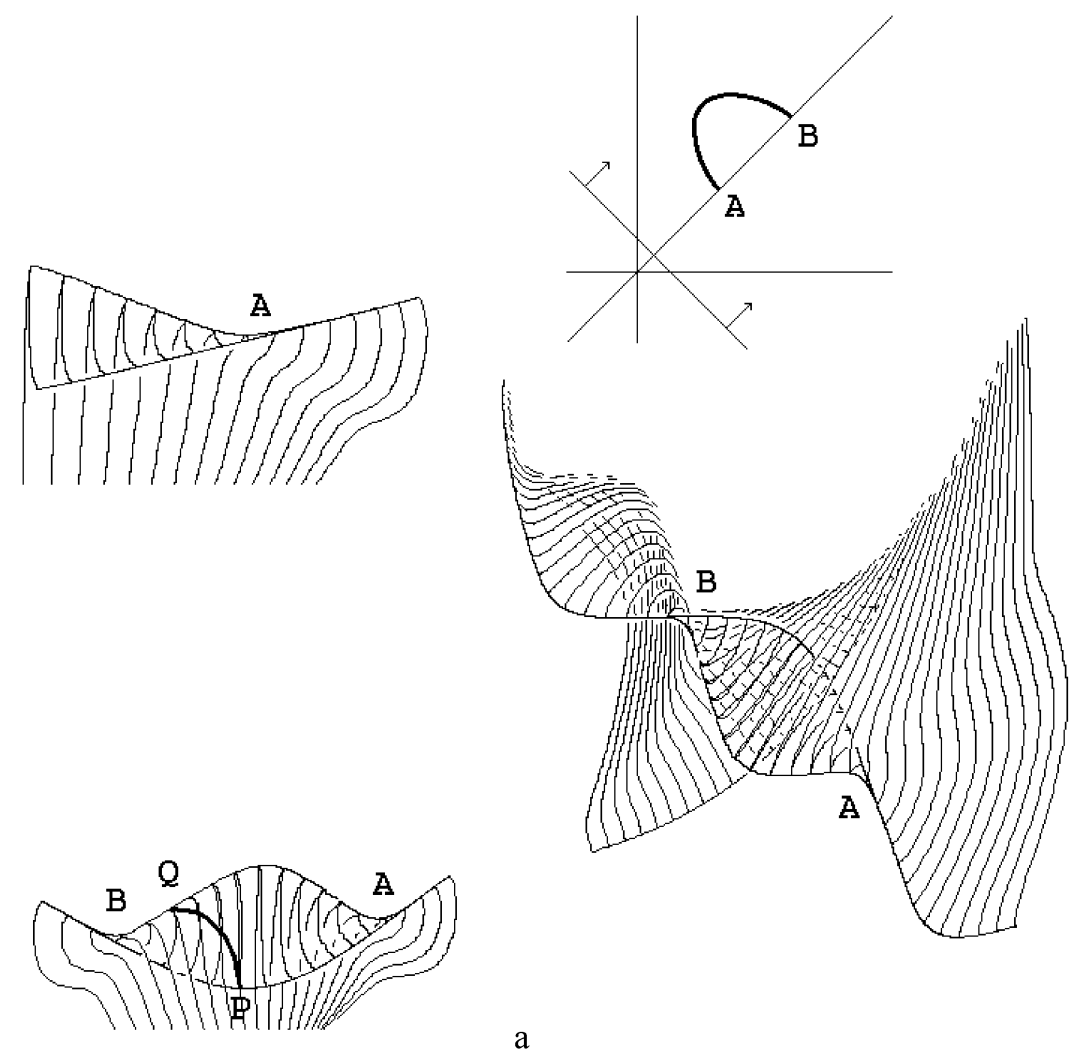

Рис. 7. Эволюция кинков.

параметры $\sigma_{1}$ и $\sigma_{2}$ увеличиваются до тех пор, пока $\varphi$ не достигнет $\varphi_{\mathrm{A}}$. В этот момент $\sigma_{1}$ имеет неограниченную скорость, касательная к ас горизонтальна, при этом $\sigma_{1}=\sigma_{\mathrm{A}}$. Далее вновь уменьшаем $\varphi, \sigma_{1}$ переходит на участок b. Линия bс обрывается в точке В.

Заменяя $\sigma_{1} \leftrightarrow \sigma_{2}$, получаем полный ответ.

3. В КСП $\sigma \sim Q^{0}$, точки мирового листа, лежашие на прямой $\sigma_{1}+\sigma_{2}=$ const, находятся при одном и том же времени в системе покоя. Величина $\beta$ представляет собой наклон касательной к линии кинка на плоскости параметров $\left(\sigma_{1}, \sigma_{2}\right): \beta=d \sigma_{1} / d \sigma_{2}$. Для выпуклых опорных кривых $\beta>0$, поэтому прямая $\sigma_{1}+\sigma_{2}=$ const пересекает линию кинка в одной точке. Для невыпуклых опорных кривых величина $\gamma=\beta+1$ может менять знак. Для эволюции кинка возможны несколько вариантов (рис. 7). Обсудим подробно некоторые детали рис. 7. Кинки появляются и исчезают одиночно на конце струны или парами внутри нее.

На линии кинка, соответствующей невыпуклой опорной кривой, функция $\gamma$ может обрашаться в нуль. При этом на линии кинка возникают особые точки. В частности, 

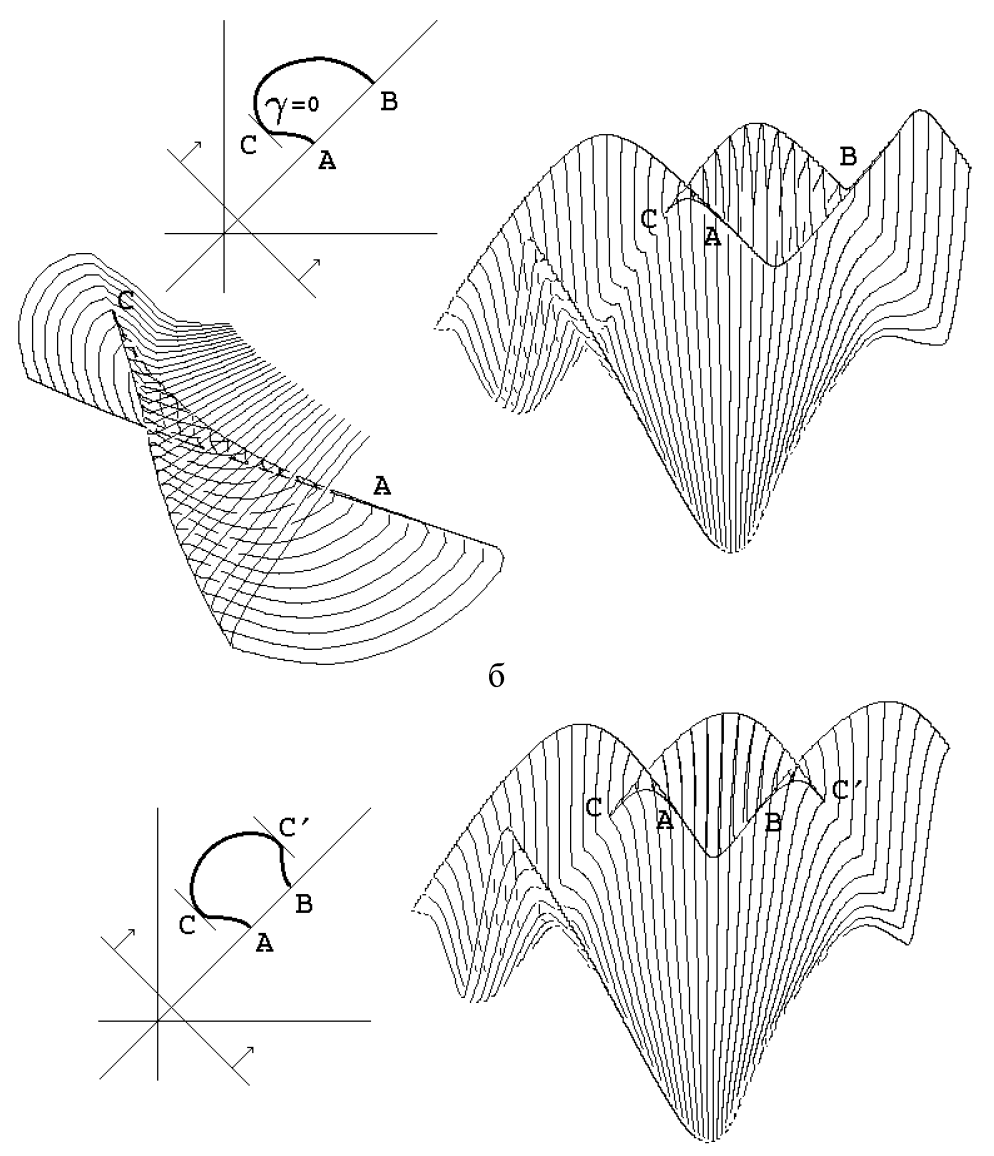

Рис. 7. Эволюция кинков (продолжение).

$\gamma=0$ на крае листа в точке перегиба (при $\left.\sigma_{1}=\sigma_{2}=\sigma_{\mathrm{A}, \mathrm{B}}\right)$, в этой точке линия кинка обрывается. Если $\gamma$ обращается в нуль во внутренних точках линии кинка (при $\sigma_{1} \neq \sigma_{2}$ ), то на линии кинка возникает точка возврата: при изменении знака $\gamma$ касательный вектор к линии кинка $Q_{1}^{\prime} d \sigma_{1}+Q_{2}^{\prime} d \sigma_{2} \sim \gamma Q_{1}^{\prime}$ меняет направление на противоположное ${ }^{5)}$. При развертке эволюции во времени в этих точках рождаются или уничтожаются пары кинков. В зависимости от числа $N$ точек возврата возможны следующие варианты эволюции кинков:

На рис. 7а $N=0$. Кинк появляется на конце струны в момент, когда конец проходит через точку перегиба А. Кинк исчезает на том же конце в момент прохождения через

\footnotetext{
${ }^{5)}$ Случай кратных нулей, когда $\gamma$ не меняет знак при прохождении через нуль, является неустойчивым.
} 


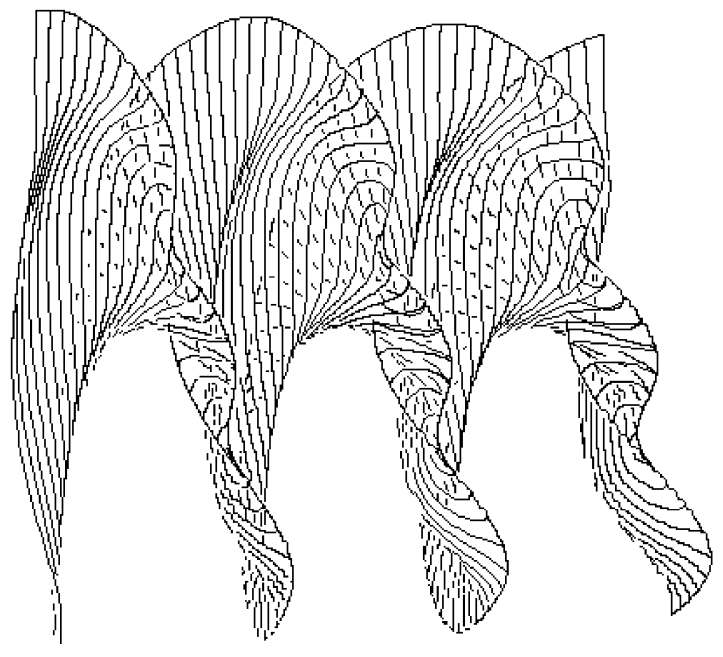

Рис. 8. Случай периодического рождения и уничтожения кинков.

точку В (на участке между точками А и В мировой лист пересекает себя по линии $P Q$ ).

На рис. 7б $N=1$. В некоторый момент времени в точке С на струне появляются два кинка. Один кинк исчезает на конце струны в точке А, другой - в точке В.

На рис. 7в $N=2$. В точке С появляется пара кинков. Один кинк исчезает на конце струны в точке А. В точке В появляется новый кинк, который уничтожает второй кинк в точке $\mathrm{C}^{\prime}$, и т.д.

Итак, при $\gamma \neq 0$ мировой лист имеет складку на линии кинка (см.рис. 3 ), случай $\gamma=0$ отвечает точкам одиночного и парного рождений кинков. Этим исчерпьваются все типы устойчивых особых точек на мировых листах в $d=3$.

4. Процессы рождения и уничтожения кинков периодически повторяются. Так, на рис. 8 сдвиги $\sigma_{1} \rightarrow \sigma_{1}+2 \pi n_{1}, \sigma_{2} \rightarrow \sigma_{2}+2 \pi n_{2}, n_{1,2} \in \mathbf{Z}$, не нарушают параллельность касательных. Кинки рождаются и исчезают на одном конце струны, затем по истечении времени, равного полупериоду $P_{0}$, эти процессы повторяются на другом конце. Они могут перекрываться во времени, новьй кинк может появиться раньше, чем исчезнет предыдущий. На струне одновременно могут находиться несколько кинков, созданных одной и той же парой точек перегиба.

5. На рис. 9 приведен случай, когда линии кинков не могут устойчиво пересекаться. При деформации опорной кривой линии кинков сближаются, касаются, затем рассеяние кинков переходит в “аннигиляционный канал”. Касание линий кинков неустойчиво (исчезает при малых деформациях опорной кривой).

6. Линии кинков на мировом листе могут образовьвать замкнутые контуры (рис. 10, пара кинков рождается в точке $\mathrm{C}$ и исчезает в точке $\mathrm{C}^{\prime}$. Для возникновения замкнутой 

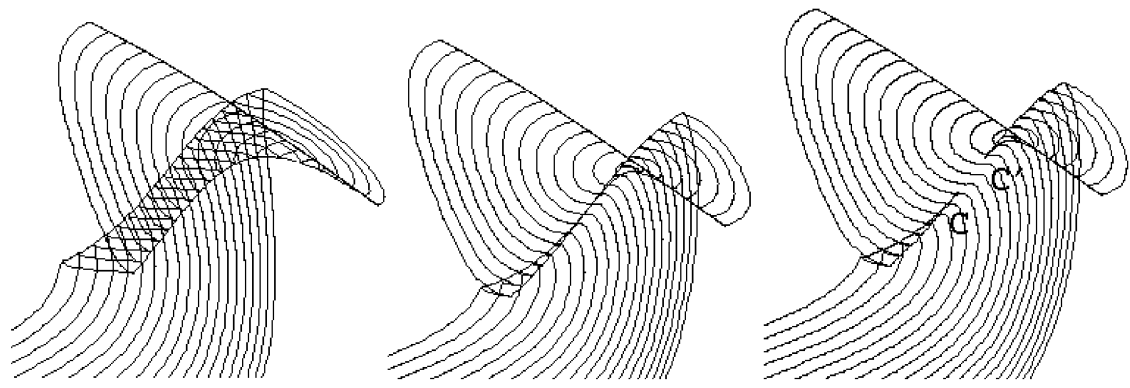

Рис. 9. Случай отсутствия устойчивого пересечения кинков.

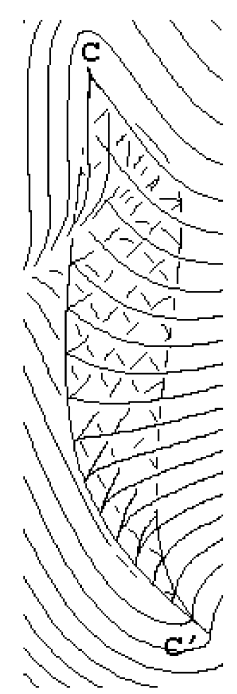

РИс. 10. Замкнутая линия кинка на мировом листе.

линии кинка необходимо, чтобы опорная кривая имела по крайней мере четыре точки перегиба).

Особые точки на мировых листах могут участвовать в более сложных процессах [7]. Все они состоят из уже описанных топологически устойчивых элементов - линий кинков и точек их одиночного и парного рождений.

\section{3. ОСОБЕННОСТИ НА МИРОВЫХ ЛИСТАХ ПРИ $d=4$ - ТОЧКИ ПИНЧА}

При $d=4$ пространство, ортогональное импульсу $P_{\mu}$, трехмерно. В КСП вектор $\vec{Q}^{\prime}(\sigma)$ лежит на двумерной сфиере. Особенности на мировом листе отвечают точкам са- 
мопересечения кривой $\vec{Q}^{\prime}(\sigma)$. Для опорных кривых общего положения особенности на мировом листе находятся в изолированных точках.

Форма мирового листа в окрестности особой точки описывается формулой (3). При этом векторы $Q_{1}^{\prime}, Q_{1}^{\prime \prime}$ и $Q_{2}^{\prime \prime}$ линейно независимы (в обшем положении), т.к. при $d=4$ пространство, ортогональное $Q_{1}^{\prime}$ и содержащее эти векторы, трехмерно:

$$
\begin{aligned}
& x\left(\sigma_{1}+\Delta \sigma_{1}, \sigma_{2}+\Delta \sigma_{2}\right)=\frac{1}{2}\left(Q_{1}+Q_{2}\right)+\frac{1}{2} Q_{1}^{\prime} \xi+\frac{1}{8 \lambda^{2}}\left(\lambda^{2} Q_{1}^{\prime \prime}-Q_{2}^{\prime \prime}\right) \xi \eta+ \\
& \quad+\frac{1}{16 \lambda^{2}}\left(\lambda^{2} Q_{1}^{\prime \prime}+Q_{2}^{\prime \prime}\right)\left(\xi^{2}+\eta^{2}\right)+o\left(\xi^{2}+\eta^{2}\right), \\
& Q_{2}^{\prime}=\lambda Q_{1}^{\prime}, \quad \xi=\Delta \sigma_{1}+\lambda \Delta \sigma_{2}, \quad \eta=\Delta \sigma_{1}-\lambda \Delta \sigma_{2} .
\end{aligned}
$$

Поверхность $(X, Y, Z)=\left(\xi, \xi \eta, \xi^{2}+\eta^{2}\right)$ изображена на рис. 11. Мировой лист в окрестности точки пинча имеет вид зонтика Уитни $[1,8]$. На рис. 11 оси образуют базис 3 -мерного изотропного пространства (касательного к световому конусу). Точно так же выглядит проекция мирового листа в окрестности изолированной особой точки в любое 3 -мерное пространство (рис. 12). Поверхность пересекает себя по линии $P Q$. В точке пинча струна претерпевает мгновенный излом. Такие особые точки называются точками пинча [8]. В этих точках пинча мировой лист не имеет касательной плоскости. При приближении к точке пинча по разным путям касательные плоскости занимают разные предельные положения.

В момент прохождения через точку пинча на струне возникает точка возврата.

ДоКАЗАТЕЛЬСТВО. В КСП $Q_{1}^{\prime \prime 0}=Q_{2}^{\prime \prime}{ }^{0}=0, \lambda=1$, поэтому на сечении поверхности (5) плоскостью $x^{0}=$ const вблизи точки пинча выполняется $\xi=$ const. В обшем положении векторы $\vec{Q}_{1}^{\prime \prime}-\vec{Q}_{2}^{\prime \prime}$ и $\vec{Q}_{1}^{\prime \prime}+\vec{Q}_{2}^{\prime \prime}$ линейно независимы. В системе координат $(Y, Z)$, натянутой на эти векторы, струна имеет вид параболы

$$
\left(\xi \eta, \xi^{2}+\eta^{2}\right)=\left(Y, \xi^{2}+Y^{2} / \xi^{2}\right)
$$

При приближении к точке пинча $\xi \rightarrow 0$, парабола вырождается в луч вдоль положительного направления оси $Z$. В этот момент струна имеет точку возврата.

В точке пинча обрьваются линии самопересечения поверхности. Заметим, что линии самопересечения имеются только на проекциях мирового листа в 3-мерное пространство. Мировой лист, изображенный на рис. 12 , в пространстве Минковского не имеет самопересечений, это видно из того, что ни одна из струн, образуюших эту поверхность, не пересекает себя. Устойчивые самопересечения мирового листа в 4-мерном пространстве Минковского располагаются в изолированных точках. 


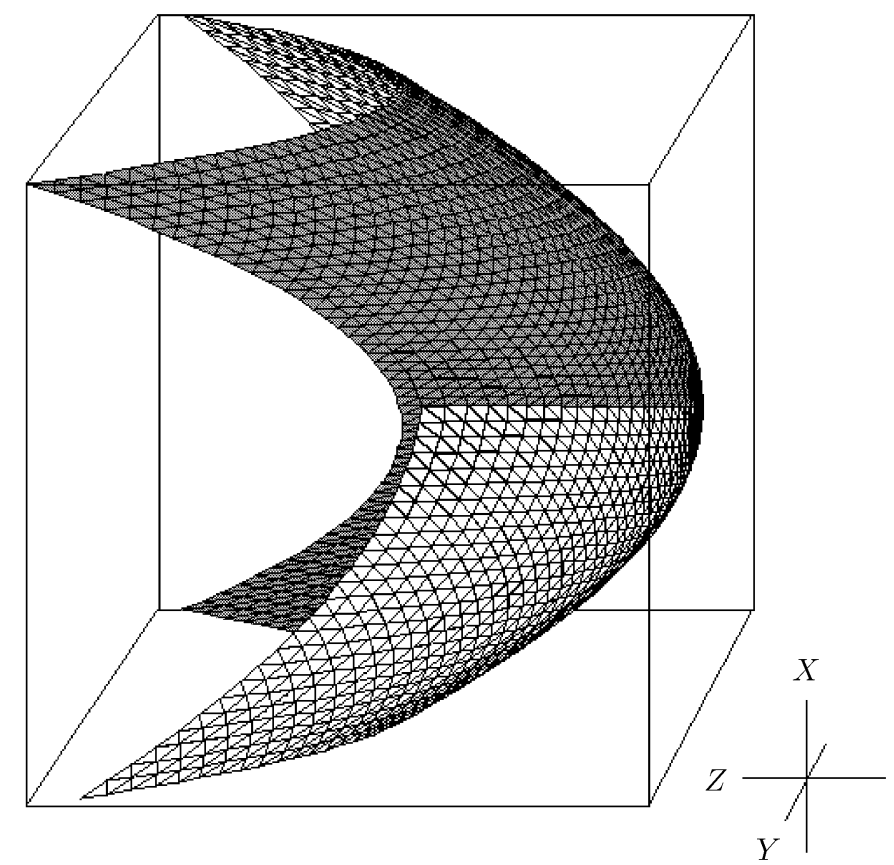

Рис. 11. Мировой лист в окрестности точки пинча.

\section{ЗАКЛЮЧЕНИЕ}

В статье проведена классификация устойчивых особых точек на мировых листах открытых струн.

При $d=3$ особенности имеют вид кинков, распространяюшихся по струне со скоростью света. Если проекция мировой линии конца струны в систему покоя струны является выпуклой кривой с числом оборотов $\nu$, то число кинков на струне сохраняется и равно $\nu-1$. Если эта кривая является невыпуклой, то на струне периодически рождаются и исчезают новые кинки одиночно на концах струны или парами внутри нее.

При $d=4$ особенности мирового листа располагаются в изолированных точках пинча. При прохождении через точку пинча струна претерпевает мгновенный излом.

При $d>4$ на мировых листах нет устойчивых особых точек.

Изображения мировых листов, приведенные в данной работе, построены с помощью комплекса графических программ [9], позволяюшего интерактивно создавать статические изображения мировых листов, а также представлять эволюцию струн в виде фильма. 


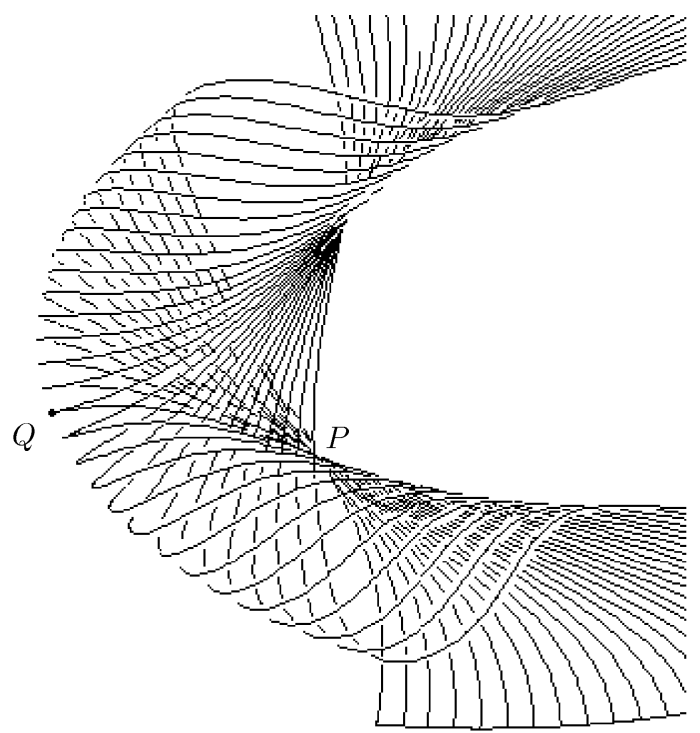

Рис. 12. Проекция мирового листа в систему покоя вблизи точки пинча $Q$.

ПРИЛОЖЕНИЕ 1

Вывод формулы (1) проведен в [10, формула (41), с. 250] (в лагранжевом подходе) ${ }^{6)}$ и в [11] (в гамильтоновом подходе). Поскольку механика струны обычно анализируется в осцилляторном представлении (см., например, [12]), мы воспроизведем доказательство формулы (1) в привычных терминах.

Фазовое пространство открытой струны описывается бесконечным набором осцилляторных переменных $a_{\mu}^{n}\left(n \in \mathbf{Z}, a_{\mu}^{n *}=a_{\mu}^{-n}\right)$ и канонической парой $X_{\mu}, P_{\mu}\left(P_{\mu}=\sqrt{\pi} a_{\mu}^{0}\right)$ со скобками Пуассона

$$
\left\{a_{\mu}^{n}, a_{\nu}^{k}\right\}=i n g_{\mu \nu} \delta^{k,-n}, \quad\left\{X_{\mu}, P_{\nu}\right\}=g_{\mu \nu}
$$

Осцилляторные переменные подчинены условиям (связям Вирасоро)

$$
L^{k}=\frac{1}{2} \sum_{n} a_{\mu}^{k-n} a_{\mu}^{n}=0 .
$$

\footnotetext{
6) При учете граничных условий для открытой струны в этой формуле $f=g$, и, кроме того, данное представление становится справедливым глобально на всем мировом листе. Соответствие с формулой (1) в нашей работе достигается при отождествлениях $f=Q / 2, a=\sigma_{1}, b=\sigma_{2}$.
} 
Кооординаты и импульсы струны определяются выражениями 7 )

$$
x_{\mu}(\sigma)=X_{\mu}+\frac{1}{\sqrt{\pi}} \sum_{n \neq 0} \frac{a_{\mu}^{n}}{i n} \cos n \sigma, \quad p_{\mu}(\sigma)=\frac{1}{\sqrt{\pi}} \sum_{n} a_{\mu}^{n} \cos n \sigma .
$$

Гамильтонианом системы является произвольная линейная комбинация связей: $H=$ $\sum c_{k} L_{k}\left(c_{k}^{*}=c_{-k}\right)$. Коэффициенты $c_{k}$ влияют лишь на параметризацию мирового листа. Выбор $H=L^{0}$ отвечает конформной параметризации (в которой $\left.\left(x^{\prime} \pm \dot{x}\right)^{2}=0\right)$. Данный гамильтониан генерирует фазовые врашения $a_{\mu}^{n}(\tau)=a_{\mu}^{n}(0) e^{i n \tau}$ и сдвиги $X_{\mu}(\tau)=$ $X_{\mu}(0)+\left(P_{\mu} / \pi\right) \tau$.

Определим функцию $Q_{\mu}(\sigma)$ выражением

$$
Q_{\mu}(\sigma)=x_{\mu}(\sigma)+\int_{0}^{\sigma} d \sigma^{\prime} p_{\mu}\left(\sigma^{\prime}\right)=X_{\mu}+\frac{P_{\mu}}{\pi} \sigma+\frac{1}{\sqrt{\pi}} \sum_{n \neq 0} \frac{a_{\mu}^{n}}{i n} e^{i n \sigma}
$$

Связи Вирасоро эквивалентны светоподобности кривой $Q_{\mu}(\sigma)$, кривая $Q_{\mu}(\sigma)$ периодична с периодом $2 P_{\mu}$.

Координаты и импульсы струны связаны с $Q_{\mu}(\sigma)$ соотношениями (2):

$$
x_{\mu}(\sigma)=\left(Q_{\mu}(\sigma)+Q_{\mu}(-\sigma)\right) / 2, \quad p_{\mu}(\sigma)=\left(Q_{\mu}^{\prime}(\sigma)+Q_{\mu}^{\prime}(-\sigma)\right) / 2 .
$$

Эволюция функции $Q_{\mu}(\sigma)$ сводится к сдвигу ее аргумента: $Q_{\mu}(\sigma, \tau)=Q_{\mu}(\tau+\sigma, 0)$. Следовательно, координаты и импульсы струны эволюционируют следуюшим образом: $x_{\mu}(\sigma, \tau)=\left(Q_{\mu}(\tau+\sigma, 0)+Q_{\mu}(\tau-\sigma, 0)\right) / 2, \quad p_{\mu}(\sigma, \tau)=\left(Q_{\mu}^{\prime}(\tau+\sigma, 0)+Q_{\mu}^{\prime}(\tau-\sigma, 0)\right) / 2$. Вводя светоподобные координаты $\sigma_{1,2}=\tau \pm \sigma$, получаем формулу (1).

\section{ПРИЛОЖЕНИЕ 2}

Если опорная кривая $Q_{\mu}(\sigma)$ является негладкой в точке $\sigma_{0}$, то мировой лист имеет излом вдоль линии

$$
x_{\mu}\left(\sigma_{0}, \sigma\right)=\frac{1}{2}\left(Q_{\mu}\left(\sigma_{0}\right)+Q_{\mu}(\sigma)\right) .
$$

На рис. 13а линия излома получается из опорной кривой сжатием в два раза к точке $\sigma_{0}$. Как показано в [7], такие кусочно-гладкие поверхности являются экстремальными. Особенности такого вида возникают при разрыве струны [5]. В отличие от линий

\footnotetext{
7) Мы положили $2 \pi \alpha^{\prime}=1$ и использовали обозначения, отличные от [12]: $a_{\mu}^{n}$ в данной работе соответствует $i \sqrt{n} a_{\mu}^{n *}$ в [12] $(n>0)$.
} 


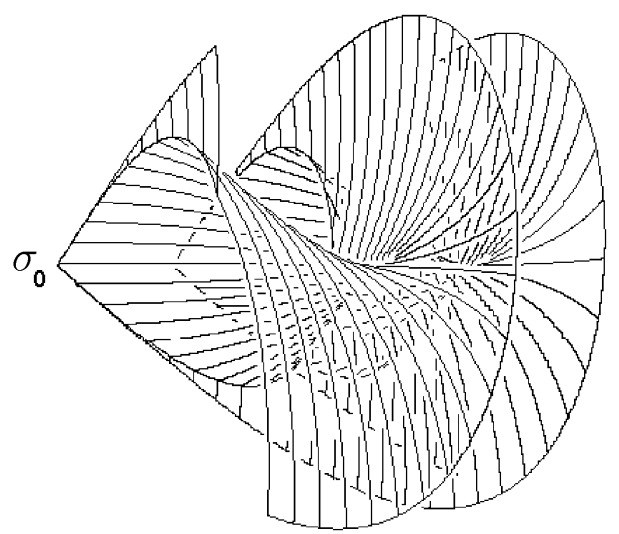

a

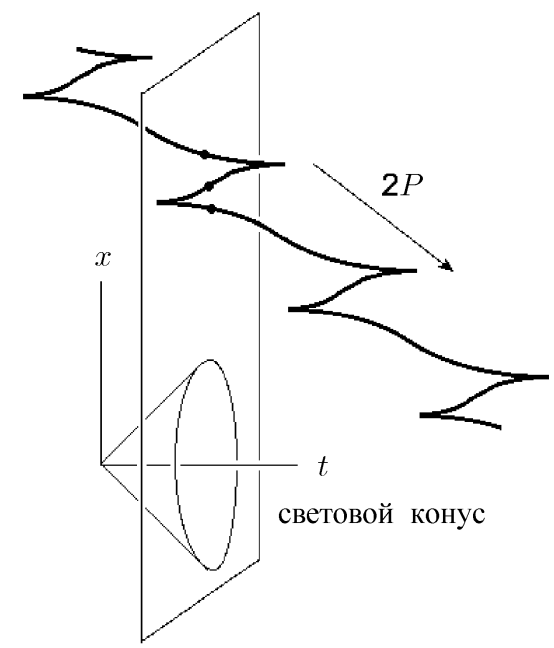

б

Рис. 13. Излом на мировом листе, вызванный негладкостью опорной кривой, (a) и опорная кривая, не монотонная по времени, (б).

кинков и точек пинча, данные особенности неустойчивы (устраняются при сглаживании опорной кривой).

В данной работе рассматриваются опорные кривые, монотонные по времени Минковского: $Q_{0}^{\prime}>0$. Заметим, что это условие не вытекает из условия светоподобности $Q^{\prime 2}=0$ (связей Вирасоро). Сушествуют светоподобные кривые, для которых $Q_{\mu}^{\prime}(\sigma)=Q_{0}^{\prime}(\sigma)(1, \vec{n}(\sigma)), \quad \vec{n}^{2}=1$ и функция $Q_{0}^{\prime}(\sigma)$ не является знакопостоянной (см. рис. 13б). В классической механике такие решения обычно исключаются из рассмот- 
рения, однако сомнения в том, что это исключение корректно проведено в квантовой теории [13], остаются.

Немонотонные опорные кривые приводят к чрезвычайно интересной механике, описанию которой мы посвятим отдельную работу.

\section{Список литературы}

[1] В.И. Арнольд, А.Н. Варченко, С. М. Гусейн-Заде. Особенности дифференцируемых отображений. Классификация критических точек, каустик и волновых фронтов. М.: Наука, 1982; М. Голубицкий, В. Гийемин. Устойчивые отображения и их особенности. М.: Мир, 1977.

[2] Г. П. Пронько и др. ЭЧАЯ. 1983. Т. 14. № 3. С. 558.

[3] Г. П. Джорджадзе, А. К. Погребков, М. К. Поливанов. ТМФ. 1979. Т. 40. № 2. С. 221; А. К. Погребков. ТМФ. 1980. Т. 45. № 2. С. 161.

[4] Y. Nambu. Phys. Rev. D. 1974. V. 10. № 12. P. 4262.

[5] X. Artru. Classical String Phenomenology. Preprints LPTHE 78/25, 79/8, 81/1. Orsay, France: Laboratoire de Physique Théorique et HautesEnergies, Université de Paris-Sua, 1978, 1979, 1981.

[6] И. Н. Никитин. ЯФ. 1993. Т. 56. № 9. С. 230; ТМФ. 1996. Т. 109. № 2. С. 202.

[7] S. V. Klimenko et al. Singularities on the World Sheets of Open Relativistic Strings. Preprint IHEP 95-7. Protvino: IHEP, 1995.

[8] Джс. К. Франсис. Книжка с картинками по топологии: Как рисовать математические картинки. М.: Мир, 1991.

[9] С. В. Клименко и др. Программирование. 1994. Т. 4. С. 47; S. V. Klimenko et al. Singularities on the world sheets of open relativistic strings. In: Proc. of 2nd Dagstuhl Seminar on Scientific Visualization (Dagstuhl, Germany, May, 1994). Chap. 18. Los Alamitos: IEEE Comp. Society Press, 1997.

[10] В. С. Владимиров. Уравнения математической физики. Изд. 5-е. М.: Наука, 1988.

[11] G. P. Pronko. Rev. Math. Phys. 1990. V. 2. № 3. P. 355.

[12] Л. Бринк, М. Энно. Принципы теории струн. М.: Мир, 1991.

[13] И. Н. Никитин. ТМФ. 1996. Т. 107. № 2. С. 213.

Поступила в редакцию 28.IV.1997 г., после доработки 24.XI.1997 г. 